\title{
Relación entre la motivación de logro académico, la autoeficacia y la disposición para la realización de una tesis
}

Fernando Ruiz Dodobara

El propósito de este estudio es dar información acerca de la problemática de la realización de tesis para obtener la licenciatura. Para ello se relacionan tres variables: la Motivación de Logro Académico, la Autoeficacia para la Realización de una Tesis y la Disposición para la Realización de una Tesis. Se tomó una muestra de estudiantes de ambos sexos pertenecientes a tres universidades privadas de Lima. Finalmente, se discute las relaciones entre las variables y su intensidad.

Motivación de logro académico / autoeficacia / disposición para escribir una tesis

Relationship between academic achievement motivation, self-efficacy, and the attitude to write a thesis

This paper is the report of a study about the problems which psychology students have to resolve when they write a thesis for obtaining the degree. In the frame of this study three variables were considered: academic achievement motivation, selfevaluated autoefficacy for writing a thesis, and the will to write her. A sample of'psychology students in the last academic semesters of three privates universities answered the psychometric reactives used for this study. The author comments the relationships between the variables and their intensity

Academic achievement motivation / self / evaluated efficacy for writing a thesis / disposition to write a thesis 
En la época actual, cuando el conocimiento y la información posibilitan avances significativos y el cambio constante, la única forma de integrarse al panorama mundial es mediante la producción del conocimiento propio o adaptado a nuestra realidad. Para conseguir lo anterior se requiere un intenso trabajo en el área de la investigación.

Al ser la universidad la institución encargada de la educación superior y de fomentar la difusión y creación del conocimiento, esta debe tener como uno de sus fines últimos la promoción y desarrollo de investigaciones exitosas que conlleven la renovación de los contenidos académicos y el impulso del progreso.

Dado que el medio universitario se caracteriza por su complejidad académica, una intensa dedicación y motivación en el estudio son necesarias para un exitoso rendimiento. Puesto que en él se fomenta la investigación mediante los múltiples cursos teórico-prácticos que se dictan, una consecuencia lógica, si es que se tiene alta motivación de logro académico, sería la realización de una tesis de investigación como culminación de los estudios de pregrado. No obstante, en los últimos años, desde la promulgación del decreto ley 739 en 1991, muchos estudiantes optan por otras modalidades de titulación, como el examen de suficiencia profesional. De esta manera, numerosas veces se produce una contradicción entre lo enseñado en el pregrado y la modalidad de titulación seleccionada.

El presente estudio intenta proporcionar información acerca de la problemática de la producción de tesis de investigación para obtener el título universitario. Con este propósito, se ha propuesto el estudio de la relación entre tres variables: la Motivación de Logro Académico, la Autoeficacia para la Realización de una Tesis y la Disposición para la Realización de una Tesis. Se quiere ver, así, si la importancia y la facilidad para la realización de una tesis guardan relación con la motivación para un rendimiento satisfactorio en el pregrado y la creencia en la capacidad para realizar la tesis exitosamente. Debemos señalar que en la presente investigación se trabajará con los aportes teóricos de McClelland y Bandura en lo referente a la Motivación de Logro y la Autoeficacia, respectivamente.

Consecuentemente, el problema del presente estudio quedó enunciado con la siguiente interrogante: ¿qué relación existe entre la Motivación de Logro Académico, la Autoeficacia y la Disposición para la Realización de una Tesis?

\section{MARCO TEÓRICO}

\section{Motivación de Logro}

La Motivación de Logro ha sido definida por Atkinson como "la disposición relativamente estable de buscar el éxito o el logro". Según McClelland "es la tendencia a alcanzar el éxito en situacio- 
nes que suponen la evaluación del desempeño de una persona, en relación con estándares de excelencia" (De Santamaría, 1991). Los estándares de excelencia pueden estar en relación con la tarea (desempeñar una tarea con éxito), con uno mismo (rendir cada vez mejor y más) o con los demás (rendir más y mejor que los otros) (Barberá y Molero, 1996).

\section{Motivación de Logro y el ámbito académico}

La Motivación de Logro posee un efecto determinante sobre los estudiantes en general $\mathrm{y}$, por ende, en las conductas que utilizan o no para desempeñarse adecuadamente en el ámbito académico.

Según Covington, existen tres tipos de estudiantes: los orientados al dominio, los que evitan el fracaso y los que aceptan el fracaso. Los primeros, con una alta Motivación de Logro, normalmente tienen éxito y se consideran a sí mismos personas capaces. También son propensos a resolver problemas y asumir riesgos que supongan retos moderados. Además, se desenvuelven adecuadamente en situaciones competitivas, aprenden con rapidez, aceptan responsabilidades con facilidad y son más persistentes ante el fracaso. Además, tienen más confianza en sí mismos, muestran más energía, requieren retroalimentación concreta y buscan captar la manera en que funciona su entorno para ser exitosos. Por su lado, los estudiantes que evitan el fracaso no están seguros acerca de su propia aptitud y buscan proteger su imagen del fracaso exagerando todo, planteándose metas muy altas o muy bajas, o realizando solo pequeños esfuerzos. Sin embargo, las estrategias para evitar el fracaso conllevan al fracaso mismo y los individuos comienzan a creer que este se debe a su falta de capacidad, convirtiéndose en estudiantes que aceptan el fracaso (Woolfolk, 1996).

La Motivación de Logro ejerce una influencia importante sobre el aprendizaje y la retención: dos elementos clave para el satisfactorio rendimiento de un estudiante. En lo concerniente al aprendizaje, el efecto catalizador de la motivación se da por medio del aumento de la atención. Según Baker y Madell, los estudiantes universitarios con un alto rendimiento académico son menos propensos a la distracción que los que poseen un bajo rendimiento. A su vez, Werner, Johnson y Merabian agregan que los alumnos con alta Motivación de Logro ensayan y piensan más sobre los problemas omitidos que los estudiantes con baja Motivación de Logro. Finalmente, hay que señalar que un nivel de motivación moderado es el que produce resultados excelentes en el aprendizaje (Ausubel, 1995).

El éxito en la vida universitaria tiene su base en la selección adecuada de una carrera acorde con las habilidades e intereses del estudiante, y se ve influenciado por la Motivación de Logro. 
Mahoma, en un estudio sobre la relación entre la elección vocacional y la motivación de temor al fracaso, llevado a cabo con 155 universitarios hombres, encontró que un número significativamente mayor de individuos con alta Motivación de Logro eligió su carrera tras una estimación realista de sus habilidades e intereses, lo opuesto ocurrió con los individuos con alta ansiedad con relación al logro (De Santamaría, 1991).

\section{Autoeficacia}

La autoeficacia puede ser definida como la percepción personal de las capacidades propias en una situación determinada. Las creencias de autoeficacia presentan gran influencia en el ser humano, ya que actúan sobre sus pensamientos, sentimientos y comportamientos (Bandura, 1995). Un aspecto que resalta la importancia de la autoeficacia es su valor predictivo de la conducta humana.

El comportamiento de las personas, según Bandura, puede ser mejor predicho por las creencias que los individuos tienen acerca de sus propias capacidades que por lo que en verdad pueden hacer, puesto que estas percepciones contribuyen a delinear qué es lo que las personas hacen con las habilidades y el conocimiento que tienen (Pajares \& Schunk, 2001). El concepto de autoeficacia se ubica dentro de la teoría social cognitiva de Bandura, la que establece una imagen del ser humano como un individuo en búsqueda del desarrollo personal. Bandura sostuvo que lo que las personas piensan sobre ellos es clave en el ejercicio del control y en la agencia humana. Aquí surge el concepto de autoeficacia, puesto que lo que las personas piensan sobre sus propias capacidades, lo que Bandura llamó autoeficacia, es el mejor predictivo de la manera en que estas personas se comportarán. Las personas son, pues, entendidas como productores y productos de sus ambientes y sistemas sociales (Pajares, 2002).

\section{Influencia de la autoeficacia en el ámbito académico}

La autoeficacia juega un papel fundamental en el ámbito académico. Se ha comprobado, mediante diversos estudios, que los conocimientos y la habilidad, de por sí, no son suficientes para garantizar y predecir un buen desenvolvimiento académico. Dos personas con la misma habilidad pueden presentar un desempeño diferente, producto de sus creencias de eficacia. Esto debido a que el éxito académico demanda procesos reguladores, como la autoevaluación, el automonitoreo y el uso de estrategias metacognitivas de aprendizaje, procesos que son influidos positivamente por un alto grado de creencia en la propia capacidad (Pajares \& Schunk, 2001).

Respecto de la motivación académica, Bandura plantea que las creencias de autoeficacia influyen en el nivel de esfuerzo, persistencia y la elección de 
actividades. Los alumnos con alto sentido de eficacia para cumplir tareas educativas persistirán más ante las dificultades, trabajarán con mayor intensidad y participarán más que aquellos que duden de sus capacidades.

Existe otra relación entre la autoeficacia y la motivación académica. Esta se da por medio de la elección de actividades. Bandura plantea que los estudiantes con alta creencia en sus capacidades elegirán tareas difíciles y desafiantes, a diferencia de sus pares con baja autoeficacia, que tenderán a evitarlas.

Hay que resaltar que la evaluación y la creación de la autoeficacia están influidas por comparaciones sociales, y en el medio universitario esto ocurre con mucha frecuencia. En este los desempeños están supeditados, en buena parte, al modelamiento y a la evaluación comparativa. Por consiguiente, los éxitos y fracasos de los demás pueden afectar la motivación personal, en tanto se parezcan a nosotros (Zimmerman, 1995).

Respecto de los profesores, la autoeficacia percibida para enseñar efectivamente tiene un impacto importante en el desempeño académico. Los profesores seguros de su capacidad para enseñar crean experiencias que aumentan la creencia de control de sus alumnos, desarrollan los intereses intrínsecos de los estudiantes y la capacidad para autodirigirse. En contraste, los profesores con baja creencia en su capacidad para enseñar generan ambientes negativos y deterioran la autoefica- cia y el desarrollo cognitivo de sus alumnos (Bandura, 1995).

Centrándonos ahora en los efectos de la autoeficacia sobre los procesos autorreguladores, debemos resaltar esta influencia sobre dos procesos en especial: el automonitoreo y el manejo y planeamiento del tiempo. El automonitoreo implica un proceso selectivo en el que nuestras creencias personales influyen en los aspectos de nuestro desempeño a los cuales damos más atención, cómo son percibidos estos y cómo es organizada la información del desempeño.

Las creencias de eficacia pueden impulsar a las personas a predecir eventos que los afectan y a buscar la manera de controlarlos. Britton y Tesser comprobaron esto mediante una investigación con universitarios centrada en el control de su tiempo. Se identificó un factor de autoeficacia que implicaba el sentimiento de creerse capaz de organizar el tiempo personal (Zimmerman, 1995).

En lo concerniente al planteamiento de metas y autoeficacia encontramos que la autoeficacia y el desarrollo de habilidades son más intensos en estudiantes que se plantean metas próximas que en aquellos que se imponen metas más distantes; las metas próximas proporcionan evidencia de una creciente destreza.

Además, los estudiantes que hayan sido motivados verbalmente a plantearse sus propias metas aumentarán su competencia, confianza y compromiso para cumplirlas. Se produce, también, 
una elevación de la autoeficacia cuando se proporciona a los estudiantes constante e inmediata retroalimentación mientras trabajan en tareas académicas.

Por otro lado, si se instruye a los alumnos en que esta retroalimentación se debe a su esfuerzo, estos trabajarán más intensamente, declararán más eficacia para próximos aprendizajes y tendrán una motivación más fuerte (Pajares \& Schunk, 2001).

\section{MetodologíA}

\section{Muestra}

La muestra se obtuvo por un procedimiento intencional no probabilístico, de las facultades de psicología de tres universidades privadas de Lima en el semestre 2003-2. Dicha muestra estuvo compuesta por 75 alumnos de psicología, entre 21 y 50 años, de tres universidades de Lima, para una población de 1.077 estudiantes de la especialidad.

Los criterios de inclusión fueron los siguientes: estar matriculados en la Facultad de Psicología de una de las tres universidades privadas del estudio, estar presentes en las aulas los días en que se realizó la evaluación, pertenecer al octavo ciclo o a un ciclo superior.

Tabla 1

\section{Composición de la muestra}

\begin{tabular}{lccc}
\hline Número & Univ. 1 & Univ. 2 & Univ. 3 \\
\hline Varones & 6 & 0 & 4 \\
Mujeres & 15 & 27 & 23 \\
Total & 21 & 27 & 27 \\
& & & \\
\hline
\end{tabular}

\section{Instrumentos}

Se construyeron tres pruebas para el presente estudio: la prueba de Motivación de Logro Académico (MLA), la prueba de Autoeficacia para la Realización de una Tesis (ART) y la prueba de Disposición para la Realización de una Tesis (DRT). Todas las pruebas fueron sometidas a jueces y obtuvieron validez de contenido.

Mediante el coeficiente de confiabilidad Alpha de Cronbach las tres pruebas obtuvieron puntajes de 0.88 (MLA), 0.90 (ART) y 0.92 (DRT), lo que indicó la conformación de tres escalas completas con puntajes confiables. Posteriormente se pasó al análisis factorial de las escalas, obteniéndose determinados factores para cada una de ellas, los cuales después de un análisis cualitativo de los ítems fueron definidos de la siguiente manera:

- Escala de Motivación de Logro.Evalúa la tendencia constante a buscar el éxito en situaciones académicas en el medio universitario. La escala obtuvo seis factores:

- Factor Búsqueda Activa del Éxito Académico. Se refiere a la acción continua de buscar un buen desempeño en situaciones académico-universitarias de evaluación de acuerdo con estándares de excelencia (19 ítems).

- Factor Retroalimentación. Hace referencia al interés por la información sobre el rendimiento de una conducta. Con esto se pre- 
tende conocer cuáles fueron las claves del éxito (15 ítems).

- Factor Originalidad. Está referido a la búsqueda de nuevas formas de realizar actividades que conduzcan a un objetivo deseado (16 ítems).

- Factor Atribución Personal del Éxito y Regulación de Tareas. Se centra en la convicción de que el éxito se debe a esfuerzos personales y a la regulación constante de las tareas propuestas. El logro proviene del sujeto, no del exterior (12 ítems).

- Factor Tareas de Dificultad Moderada. Evalúa la predisposición a emprender tareas de dificultad intermedia, ya que si se trata de tareas fáciles cualquiera las puede realizar; si, por otro lado, son tareas difíciles, la mayoría falla en el intento (6 ítems).

- Factor Metas Altas Realistas. Observa la preferencia por las metas de alto nivel de exigencia dentro del rango de capacidad de la persona (14 ítems).

- Escala de Autoeficacia para la Realización de una Tesis.- Evalúa la creencia en la capacidad personal para realizar una tesis. La escala obtuvo dos factores:

- Factor Autoeficacia PersonalSocial. Evalúa la capacidad percibida para realizar una tesis, superando impedimentos produci- dos por estados de ánimo negativos, falta de apoyo del entorno social y distractores (10 ítems).

- Factor Autoeficacia Académica. Se centra en la capacidad percibida para superar los obstáculos académicos propios de las exigencias de la realización de una tesis (4 ítems).

- Escala de Disposición para la Realización de una Tesis. Evalúa la importancia que se confiere a la tesis y la facilidad en general para realizarla. La escala obtuvo cuatro factores:

- Factor Importancia en la Formación Académica. Evalúa la importancia que una persona le da a la tesis en su formación académica (17 ítems).

- Factor Facilidades Académicas. Se centra en el grado en que el medio universitario promueve la realización de una tesis, poniendo a disposición de los postulantes los profesionales (asesores) y recursos académicos necesarios (14 ítems).

- Factor Utilidad Futura. Hace referencia a la consideración de la tesis como medio para alcanzar futuros logros profesionales (académicos, laborales) (6 ítems).

- Factor Confianza Académica para la Tesis. Se refiere a la seguridad que se tiene en la propia formación teórico-metodológica para realizar una tesis (6 ítems). 


\section{Procedimientos}

Los datos fueron recolectados en los meses de octubre y noviembre del 2003. Para esto se aplicaron las tres pruebas elaboradas: Motivación de Logro Académico (MLA), Autoeficacia para la Realización de una Tesis (ART) y Disposición para la Realización de una Tesis (DRT), una a continuación de otra.

Los instrumentos de investigación (pruebas de MLA, ART y DRT) fueron administrados en tres universidades privadas de manera grupal y en las propias aulas de clase de los estudiantes.

\section{Resultados}

Se debe resaltar que siete casos fueron eliminados al obtener una puntuación mayor o igual de seis puntos en una escala de Deseabilidad Social-Mentiras (Alpha de Cronbach: 0.65). La eliminación se realizó por la contaminación de sus respuestas, con mentiras o deseos de dar una imagen socialmente aceptable.

\section{Análisis descriptivo}

Se procedió a realizar el análisis Kolmogorov-Smirnov a los datos obtenidos en las tres pruebas. Se apreció que las puntuaciones obtenidas presentaban valores que no eran estadísticamente significativos, por lo que pudimos concluir que se ajustaban a la distribución de la curva normal. En consecuencia, se decidió utilizar estadísticos paramétricos para el contraste de las hipótesis del presente estudio.

\section{Correlación entre variables}

En primer lugar, apreciamos la inexistencia de una correlación positiva entre la Motivación de Logro Académico y la Autoeficacia para la Realización de una Tesis. Esto queda graficado en la tabla

Tabla 2

Matriz de correlaciones entre la Motivación de Logro Académico y la Autoeficacia para la Realización de una Tesis

\begin{tabular}{lccc}
\hline Correlación & $\begin{array}{c}\text { Autoeficacia } \\
\text { Personal-Social }\end{array}$ & $\begin{array}{c}\text { Autoeficacia } \\
\text { académica }\end{array}$ & Total ART \\
\hline Búsqueda de Éxito Acad. & -0.1 & -0.01 & -0.06 \\
Retroalimentación & 0.7 & 0.19 & 0.14 \\
Originalidad & $0.24^{*}$ & 0.11 & 0.20 \\
Atribución Personal del Éxito y & & & \\
$\quad$ Regulación de Tareas & 0.12 & 0.20 & 0.18 \\
Tareas de Dificultad Moderada & $0.25^{*}$ & $0.30^{*}$ & $0.31^{* *}$ \\
Metas Altas Realistas & 0.08 & 0.22 & 0.17 \\
Total MLA & 0.14 & 0.21 & 0.20 \\
\hline
\end{tabular}

${ }^{*} p<0,05 \quad{ }^{* *} p<0,01 \quad N=68$ 
2, donde se observa un bajo coeficiente de Pearson no significativo de 0.20 .

Se debe añadir que el factor de la Motivación de Logro Académico, Tareas de Dificultad Moderada, muestra una correlación positiva de 0.31 , significativa a un $\mathrm{p}<0.01$ con la totalidad de la Autoeficacia para la Realización de una Tesis. Específicamente, se encuentran correlaciones positivas entre el factor Tareas de Dificultad Moderada y los factores Autoeficacia Personal Social (0.25) y Autoeficacia Académica (0.30): ambas son significativas a un $\mathrm{p}<0.05$.

En segundo lugar podemos observar la existencia de una relación positiva entre la Motivación de Logro Académico y la Disposición para Realización de una Tesis, graficada en la tabla 3. Aquí se encuentra que el total de Motivación de Logro Académico y el total de Disposición para la Realización de una Tesis alcanzan una correlación positiva de 0.45 que es significativa a un $p<0.01$, la cual puede clasificarse como moderada.

Cabe señalar que el factor de Confianza Académica para la Tesis, de la Disposición para la Realización de una Tesis, guarda correlaciones positivas significativas a un $\mathrm{p}<0.01$ con todos los factores de la Motivación de Logro Académico. Estas correlaciones varían desde 0.39 con el factor Búsqueda de Éxito Académico hasta 0.58 con el total de la Motivación de Logro Académico.

Otro factor de la Disposición para la Realización de una Tesis, que presenta correlaciones positivas significativas

Tabla 3

Matriz de correlaciones entre la Motivación de Logro Académico y la Disposición para la Realización de una Tesis

\begin{tabular}{|c|c|c|c|c|c|c|c|}
\hline \multirow[t]{2}{*}{ Correlación } & \multicolumn{7}{|c|}{ Motivación de Logro Académico } \\
\hline & 1 & 2 & 3 & 4 & 5 & 6 & 7 \\
\hline \multicolumn{8}{|c|}{ Importancia en la Formación } \\
\hline Académica & $0.35^{* *}$ & $0.26^{*}$ & 0.21 & $0.34^{* *}$ & $0.29^{*}$ & $0.31^{* *}$ & $0.37^{* *}$ \\
\hline Facilidades Académicas & 0.20 & 0.02 & 0.11 & 0.21 & 0.18 & $0.27^{*}$ & 0.21 \\
\hline Utilidad Futura & 0.10 & 0.17 & 0.05 & 0.17 & 0.03 & 0.23 & 0.16 \\
\hline $\begin{array}{l}\text { Confianza Académica } \\
\text { para la Tesis }\end{array}$ & $0.39^{* *}$ & $0.41^{* *}$ & $0.56^{* *}$ & $0.46^{* *}$ & $0.36^{* *}$ & $0.57^{\star *}$ & $0.58^{* *}$ \\
\hline Total DRT & $0.36^{\star *}$ & $0.29^{*}$ & $0.32^{* *}$ & $0.41^{* *}$ & $0.29^{*}$ & $0.48^{* *}$ & $0.45^{* *}$ \\
\hline
\end{tabular}

${ }^{*} p<0.05 \quad{ }^{* *} p<0.01 \quad N=68$

Nota: 1) Búsqueda de Éxito Académico, 2) Retroalimentación, 3) Originalidad, 4) Atribución Personal del Éxito y Regulación de Tareas, 5) Tareas de Dificultad Moderada, 6) Metas Altas Realistas, 7) Total de Motivación de Logro Académico. 
Tabla 4

Matriz de correlaciones entre la Autoeficacia para la Realización de una Tesis y la Disposición para la Realización de una Tesis

\begin{tabular}{lccc}
\hline Correlación & $\begin{array}{c}\text { Autoeficacia } \\
\text { personal-social }\end{array}$ & $\begin{array}{c}\text { Autoeficacia } \\
\text { académica }\end{array}$ & Total ART \\
\hline Importancia de la Formación Académica & -0.02 & 0.08 & 0.03 \\
Facilidades Académicas & -0.14 & 0.11 & -0.02 \\
Utilidad Futura & -0.19 & -0.01 & -0.11 \\
Confianza Académica para la Tesis & 0.18 & $0.28^{*}$ & $0.26^{*}$ \\
Total DRT & -0.07 & 0.16 & 0.04 \\
\hline
\end{tabular}

${ }^{*} p<0.05 N=68$

con casi todos los factores de la Motivación de Logro Académico menos con el factor Originalidad, es el factor Importancia en la Formación Académica. Este factor muestra una correlación positiva de 0.37 significativa a un $\mathrm{p}<$ 0.01 con el total de Motivación de Logro Académico, además de correlaciones positivas significativas que varían desde 0.26 a un $p<0.05$ con el factor Retroalimentación hasta 0.35 significativa a un $\mathrm{p}<0.01$ con el factor Búsqueda de Éxito Académico.

En tercer lugar, verificamos la inexistencia de una correlación positiva entre la Autoeficacia para la Realización de una Tesis y la Disposición para la Realización de una Tesis. Esto queda evidenciado en la tabla 4, en la que observamos un bajo Coeficiente de Pearson de 0.04 .

No obstante, debemos señalar que existe una correlación positiva entre el factor Confianza Académica para la Tesis y la totalidad de la Autoeficacia para la Realización de una Tesis de 0.26 que es significativa a un $\mathrm{p}<0.05$. Específicamente, observamos una correlación positiva de 0.28 significativa a un $\mathrm{p}<0.05$ entre el factor Confianza Académica para la Tesis y el Factor Autoeficacia Académica.

En la tabla 5 se pueden apreciar los resultados del análisis de regresión múltiple, donde el total de la Motivación de Logro Académico y el total de la Autoeficacia para la Realización de una Tesis permiten predecir la Disposición para la Realización de una Tesis.

Los hallazgos indican que el resultado es estadísticamente significativo $(\mathrm{F}=8.53, \mathrm{p}<0.01)$, alcanzándose una correlación múltiple entre las tres variables de 0.46 que es significativa a un $\mathrm{p}<0.01$.

Cabe destacar que el mayor peso viene presentado por la Motivación de Logro Académico, con un 0.46, mientras que la Autoeficacia para la Rea- 
Tabla 5

Resumen del Análisis de Regresión Múltiple del total de Motivación de Logro Académico y el total de Autoeficacia sobre la Disposición para Realizar una Tesis

\begin{tabular}{|c|c|c|c|c|}
\hline \multirow[t]{2}{*}{ Variables independientes } & \multicolumn{2}{|c|}{$\begin{array}{l}\text { Coeficientes } \\
\text { no estandarizados }\end{array}$} & \multirow{2}{*}{$\begin{array}{l}\text { Coeficientes } \\
\text { estandarizados } \\
\text { Beta }\end{array}$} & \multirow[t]{2}{*}{$\mathbf{t}$} \\
\hline & B & Error típ. & & \\
\hline (Constante) & 2.34 & 0.44 & & $5.28^{* * *}$ \\
\hline Total Motivación de Logro & 0.49 & 0.12 & 0.46 & $4.11^{* * *}$ \\
\hline Total Autoeficacia & 0.03 & 0.07 & 0.05 & -0.44 \\
\hline $\mathbf{F}$ & $8.53^{* *}$ & & & \\
\hline $\mathbf{R}$ & $0.46^{* *}$ & & & \\
\hline $\mathbf{R}^{2}$ & 0.21 & & & \\
\hline
\end{tabular}

lización de una Tesis tiene un peso menos selectivo de 0.05 .

\section{Intensidad de las variables}

En la tabla 6 se observa que existen diferencias significativas entre el valor alcanzado en el total de Motivación de Logro Académico y el valor Prueba, que en este caso es de $3(\mathrm{t}=13.31 ; \mathrm{gl}=$ $67 ; \mathrm{p}<0.001)$. Debido a que el total de Motivación de Logro Académico alcanzó un puntaje de 3.61, se puede concluir que se alcanzó un nivel superior al moderado.

Debemos agregar que en el análisis de los componentes se aprecia la exis-

Prueba t de Student de comparación de medias para una muestra de las áreas de la escala para evaluar la Motivación de Logro Académico

\begin{tabular}{lccc}
\hline & $\mathbf{M}$ & $\mathbf{D E}$ & $\mathbf{T}$ \\
\hline Búsqueda de Éxito Acad. & 3.53 & 0.50 & $8.12^{\star * *}$ \\
Retroalimentación & 3.57 & 0.49 & $9.57^{\star * \star}$ \\
Originalidad & 3.39 & 0.46 & $6.99^{\star * *}$ \\
Atribución Personal del Éxito-Reg. de Tareas & 3.74 & 0.42 & $14.66^{\star * \star}$ \\
Tareas de Dificultad Moderada & 3.68 & 0.52 & $10.78^{\star * \star}$ \\
Metas Altas Realistas & 3.77 & 0.51 & $12.48^{\star * *}$ \\
Total MLA & 3.61 & 0.38 & $13.31^{\star * *}$ \\
\hline
\end{tabular}

\footnotetext{
${ }^{* * *} \mathrm{p}<0.001$ Valor de prueba $=3$
}

$\mathrm{N}=68$ 
tencia de diferencias significativas en Búsqueda de Éxito Académico $(\mathrm{t}=8.21$; $\mathrm{gl}=67 ; \mathrm{p}<0.001)$, Retroalimentación ( $\mathrm{t}$ $=9.57 ; \mathrm{gl}=67 ; \mathrm{p}<0.001)$, Originalidad $(\mathrm{t}=6.99, \mathrm{gl}=67 ; \mathrm{p}<0.001)$, Atribución Personal del Éxito-Regulación de Tareas $(\mathrm{t}=14.66, \mathrm{gl}=67 ; \mathrm{p}<0.001)$, Tareas de Dificultad Moderada $(\mathrm{t}=$ 10.78; $\mathrm{gl}=67 ; \mathrm{p}<0.001)$, Metas Altas Realistas ( $\mathrm{t}=12.48 ; \mathrm{gl}=67 ; \mathrm{p}<0.001)$. Se evidencia, así, que en todos los casos el valor observado es superior al valor de prueba.

A continuación encontramos que la Autoeficacia para la Realización de una Tesis no supera el nivel moderado, ya que observamos (tabla 7) que no existen diferencias significativas $(\mathrm{t}=$ 1.84; $\mathrm{gl}=67 ; \mathrm{p}<0.05)$, con lo cual concluimos, pues el valor observado $(\mathrm{M}=2.86 ; \mathrm{DE}=0.64)$ es equivalente al valor de la prueba, que es de 3 .

$\mathrm{Se}$ debe resaltar que en el factor $\mathrm{Au}-$ toeficacia Académica tampoco se observan diferencias significativas $(\mathrm{t}=$
$0.52 ; \mathrm{gl}=67 ; \mathrm{p}<0.05)$. En tanto que en el factor Autoeficacia Personal-Social sí existen diferencias estadísticamente significativas $(\mathrm{t}=3.61 ; \mathrm{gl}=67$; $\mathrm{p}<0.001$ ), ya que el puntaje es inferior al valor de prueba $(\mathrm{M}=2.67 ; \mathrm{DE}=$ 0.75 ).

También encontramos que existe un nivel superior al moderado de Disposición para la Realización de una Tesis en los estudiantes de psicología. Esto se puede corroborar en la tabla 8 , donde se observa que existen diferencias significativas entre el valor alcanzado en el total de Disposición para la Realización (4.04) de una Tesis y el valor de prueba, que en este caso es de $3(\mathrm{t}=$ 21.09; $\mathrm{gl}=67 ; \mathrm{p}<0.001)$.

Adicionalmente, en el análisis de los componentes se observa que existen diferencias significativas en Importancia de Formación Académica $(\mathrm{t}=$ 16.44; $\mathrm{gl}=67 ; \mathrm{p}<0.01)$, Facilidades Académicas $(\mathrm{t}=10.28 ; \mathrm{gl}=67 ; \mathrm{p}<$ 0.01), Utilidad Futura $(\mathrm{t}=16.19 ; \mathrm{gl}=$

Tabla 7

Prueba t de Student de comparación de medias para una muestra de las áreas de la escala para evaluar la Autoeficacia para la Realización de una Tesis

\begin{tabular}{lccc}
\hline & M & DE & T \\
\hline Autoeficacia Personal- Social & 2.67 & 0.75 & $-3.61^{\star \star *}$ \\
Autoeficacia Académica & 3.04 & 0.71 & 0.52 \\
Total ART & 2.86 & 0.64 & -1.84 \\
\hline
\end{tabular}

\footnotetext{
${ }^{* * *} p<0.001 \quad$ Valor de prueba $=3$

$\mathrm{N}=68$
} 
Tabla 8

Prueba t de Student de comparación de medias para una muestra de las áreas de la escala para evaluar la Disposición para la Realización de una Tesis

\begin{tabular}{lccc}
\hline & M & DE & T \\
\hline Importancia de Formación Académica & 4.11 & 0.54 & $16.94^{\star *}$ \\
Facilidades Académicas & 3.78 & 0.62 & $10.28^{\star *}$ \\
Utilidad Futura & 4.21 & 0.62 & $16.19^{\star * *}$ \\
Confianza Académica para la Tesis & 4.05 & 0.52 & $16.50^{\star * *}$ \\
Total DRT & 4.04 & 0.41 & $21.09^{\star * *}$ \\
\hline
\end{tabular}

${ }^{* *} p<0.01 \quad$ Valor de prueba $=3$

$\mathrm{N}=68$

$67 ; \mathrm{p}<0.001)$ y en Confianza Académica para la Tesis $(\mathrm{t}=16.50 ; \mathrm{gl}=67$; $\mathrm{p}<0.001)$.

De esta manera apreciamos que en todos los casos el valor observado es superior al valor de prueba.

\section{Discusión}

En lo que corresponde a la relación positiva entre la Motivación de Logro Académico y la Autoeficacia para la Realización de una Tesis en estudiantes de psicología de tres universidades privadas de Lima, encontramos que no existe una relación significativa. Este hallazgo resulta sorprendente, porque si una persona está motivada a buscar el éxito en situaciones académicas del pregrado, entonces, supuestamente, obtendrá los conocimientos necesarios para hacer una tesis; entendiendo la tesis como la conjunción de varios conocimientos del pregrado. Por consiguiente, con estos conocimientos obte- nidos, una persona debería creer que puede realizar una tesis, o dicho de otra manera, tener una alta autoeficacia para realizar una tesis. De igual manera, la situación inversa podría producirse. Si una persona no está motivada para rendir adecuadamente en el pregrado, entonces no obtiene los conocimientos necesarios para estructurar una tesis, por lo tanto no debería sentir que posee la capacidad para realizar una tesis, $y$, por consiguiente, debería elegir otra opción si es que desea titularse.

Se puede inferir, entonces, que estaríamos hablando de un divorcio entre la motivación para buscar el éxito en situaciones académicas del pregrado y la creencia en la capacidad de la persona o autoeficacia para realizar una tesis. Pensamos que la explicación a esta desconexión entre estas variables se encuentra relacionada con los niveles de la Motivación de Logro Académico y de la Autoeficacia para la Realización de una Tesis. 
Respecto de la existencia de una relación positiva entre la Motivación de Logro Académico y la Disposición para Realizar una Tesis en los estudiantes de psicología de tres universidades privadas de Lima, los resultados obtenidos mostraron que, efectivamente, existe una relación positiva y significativa entre ambas variables. Este hallazgo era de esperarse, ya que la Motivación de Logro Académico implica la búsqueda constante de éxito en tareas de índole académica y la tesis es una tarea de este tipo que concentra buena parte de la preparación del pregrado.

Por otro lado, cabe resaltar la relación positiva entre el factor Importancia en la Formación Académica, el total de la Motivación de Logro Académico y la casi totalidad de sus áreas. La explicación para ello estaría en el hecho de que si se considera la tesis como una tarea compleja y gravitante en la formación académica, se necesita de un alto nivel de Motivación de Logro Académico para su realización. Por consiguiente, se deben poner en práctica las estrategias implicadas en la Motivación de Logro (retroalimentación, regulación de tareas, establecimiento de metas realistas, etcétera). Este hallazgo tiene relación con los estudios realizados por Covington, en los que se comprobó que los estudiantes orientados al dominio tienen una alta Motivación de Logro y son propensos a resolver problemas académicos complejos y a asu- mir riesgos que impliquen retos moderados realistas, son más persistentes ante el fracaso, requieren retroalimentación, aprenden con rapidez y se desenvuelven bien en escenarios competitivos (Woolfolk, 1996).

Se debe llamar la atención sobre la relación positiva entre el factor Confianza Académica para la Tesis y cada una de las áreas de la Motivación de Logro Académico. Asumimos que, dado que la tesis implica una concentración de varios de los saberes del pregrado, la seguridad en tener los conocimientos necesarios para realizarla puede extenderse a la confianza en la formación académica que se tiene en general. Por lo tanto, la relación de la Motivación de Logro Académico con el componente Confianza Académica para la Tesis demostraría que la creencia en la formación académica, tanto teórica como metodológica, es primordial para estar motivado en la búsqueda del éxito académico en general. Guiándonos por los resultados, podemos concluir que esta convicción posibilitaría el planteamiento de propuestas académicas originales y la fijación de metas altas pero realistas. Además, en la medida en que se tiene confianza en los conocimientos propios, se atribuyen los logros al esfuerzo y preparación personal. Por otro lado, se podría exigir retroalimentación, ya que se cree en los propios juicios y se busca confirmarlos.

En lo concerniente a la existencia de una relación positiva entre la Auto- 
eficacia para la Realización de una Tesis y la Disposición para Realizar una Tesis en los estudiantes de psicología de tres universidades privadas de Lima, los resultados demuestran que no existe una relación significativa entre ambas variables. A pesar de que este hallazgo puede parecer contradictorio, la explicación que encontramos es que a pesar de concebir una tarea académica como importante y útil, y de poseer los recursos para realizarla (facilidades y capacidad personal), las personas pueden pensar que no tienen la capacidad suficiente para llevarla a cabo, o mejor dicho, pensar que no pueden vencer los obstáculos que les impiden realizarla. Este hallazgo guarda relación con lo encontrado en un estudio de Shell, Murphy y Bruning (1989), donde se investigó la relación entre la autoeficacia y la expectativa de resultados en el éxito para leer y escribir. La autoeficacia fue medida como la capacidad percibida para ejecutar varias actividades de escritura y lectura. Por su parte, las expectativas de resultados se midieron como la importancia de leer y escribir para conseguir resultados en empleos, recompensas sociales, educación, vida familiar y ciudadanía. Ambas variables predijeron juntas el 32 por ciento de la varianza en el éxito de la lectura; no obstante, de este porcentaje la autoeficacia predijo el 28 por ciento de la varianza. Además, solo la autoeficacia percibida se constituyó en un predictor significativo del éxito en la escritura.
Es de resaltar que el factor Confianza Académica para la Tesis obtiene correlaciones positivas y significativas con el factor Autoeficacia Académica; esto último lo hace alcanzar una correlación significativa con el total de la Autoeficacia para la Realización de una Tesis.

La explicación sería la semejanza entre ambos factores a cierto nivel, ya que mientras el factor Confianza Académica para la Tesis hace referencia a la seguridad en la formación para estructurar una tesis, el factor Autoeficacia Académica se entiende como la capacidad percibida para cumplir adecuadamente con las exigencias educativas para desarrollarla, como son la búsqueda de bibliografía y materiales como test, escalas, etcétera.

Respecto de la existencia de una relación positiva entre la Motivación de Logro Académico, la Autoeficacia para la Realización de una Tesis y la Disposición para la Realización de una Tesis se observa que hay una relación estadísticamente significativa entre las tres variables; ubicando a la Disposición para la Realización de una Tesis como variable dependiente. Cabe indicar que la relación positiva se produce, sobre todo, por la relación significativa entre la Motivación de Logro Académico y la Disposición para la Realización de una Tesis. Esto debido a que no existe una relación significativa entre la Disposición para la Realización de una Tesis y la Autoeficacia para la Realización de una Tesis. 
Con relación al nivel de Motivación de Logro Académico los resultados evidencian la existencia de un nivel superior al moderado. Por lo tanto, podríamos concluir que:

- los estudiantes, en general, buscan desempeñarse adecuadamente en situaciones de evaluación universitaria;

- están pendientes de la información sobre los efectos de su conducta para, de esta manera, buscar corregirla o mejorarla, consiguiendo así mejores resultados;

- la originalidad es un factor apreciado por los estudiantes, por lo que se infiere que buscan innovar en sus actividades (trabajos, exposiciones, etcétera);

- consideran que el éxito o fracaso en sus actividades se debe a sus propios esfuerzos y a la regulación y monitoreo de las actividades y no proviene de fuentes externas;

- prefieren tareas con un nivel de dificultad intermedio, descartando las tareas demasiado fáciles o las extremadamente complicadas.

También afirmamos que las metas que se plantean los estudiantes, si bien pueden tener un alto nivel de exigencia, no escapan de su capacidad real de concreción.

Dado que la Motivación de Logro Académico y cada uno de sus aspectos le son favorables a los estudiantes de psicología de las tres universidades, podemos aventurarnos a afirmar que su rendimiento académico es satisfactorio en general. Ya que una elevada motivación de logro posibilita un mejor desempeño académico. Esta afirmación guarda relación con la investigación planteada por Atkison y Litwin (1966), en el que se buscó relacionar la motivación de logro con la ansiedad y el éxito en los exámenes. El estudio tomó como muestra a universitarios varones estadounidenses de segundo y tercer años. Se comprobó que los estudiantes con una motivación de logro mayor elegían con más frecuencia tareas de dificultad intermedia, trabajaban durante más tiempo en un examen final y conseguían puntajes más altos que los estudiantes con menor motivación de logro (De Santamaría, 1991).

Respecto del nivel de Autoeficacia para la Realización de una Tesis, los resultados demuestran que no existe un nivel superior al moderado de Autoeficacia. Por lo tanto, podríamos afirmar que los estudiantes carecen de una fuerte creencia en su capacidad para llevar a cabo una tesis. Se podría encontrar una explicación centrándonos en el factor Autoeficacia Personal-Social, el cual obtiene puntuaciones estadísticamente significativas negativas. Esto implica que el mayor impedimento para concretar una tesis proviene del ambiente social próximo y de los estados de ánimo. Es decir, los estudiantes no se creen capaces de realizar una tesis cuando deben superar obstáculos como: sentirse depri- 
mido o ansioso; carecer de apoyo de familiares, amigos y asesor de tesis; tener compromisos sociales y actividades interesantes. Lo anterior se explica, en parte, porque una tesis, por su nivel de trabajo y complejidad, requiere organización y apoyo efectivo. Es decir, la persona debe tener un alto nivel de eficacia en la organización de su tiempo (estudio, trabajo, compromisos). Un ambiente que apoya efectivamente a la persona que está realizando una tesis posibilita una organización más eficiente de sus horarios y, por ende, incrementa la creencia del control sobre el tiempo propio, lo que permite una dedicación más intensa y efectiva a la tesis. Esto se relaciona con la investigación de Britton y Tasser (1991) sobre la autoeficacia en el control del tiempo en universitarios. La creencia en la capacidad de organizar el tiempo (estudio, compromisos sociales, trabajo) fue una mejor predictora del éxito académico que test estandarizados, cuatro años más tarde (Bandura, 1995).

Por otro lado, debemos resaltar que, pese a que no obtiene puntuaciones significativas, la del factor Autoeficacia Académica no supera el nivel moderado. Esto podría entenderse como el hecho de que los estudiantes no depositarían mucha confianza en su capacidad académica para realizar una tesis. Lo cual querría decir que no confían demasiado en su capacidad para buscar bibliografía y materiales adecuados (test, escalas), como tampoco en su capacidad para superar la presión causada por el estudio o trabajo, mientras rea- lizan una tesis. Una explicación posible para lo afirmado la encontramos en la experiencia vicaria, como forma de fortalecimiento y creación de la autoeficacia percibida. Nos referimos a que si apreciamos que personas similares a nosotros tienen éxito en determinadas tareas, entonces nuestra creencia en que podemos tener éxito en estas aumentará. De la misma manera, si se ve que personas parecidas a nosotros fracasan en una tarea, la creencia en nuestra capacidad para llevarla a cabo con éxito disminuye. Como se supone a la universidad como un medio donde reina una comparación constante, la creencia en la capacidad para desarrollar una tesis de investigación puede estar decreciendo en los estudiantes, al ver que otros similares anteriores a ellos no realizaron una tesis de investigación para obtener el título de licenciatura. Lo anterior se relaciona con un estudio llevado a cabo por Brown e Inouye (1978) con universitarios, en donde se evaluaba su eficacia para solucionar anagramas. Se indicó a los sujetos que su desempeño era mejor o igual que un modelo que fallaba la tarea. Si los sujetos pensaban que el modelo tenía menor habilidad que ellos, persistían en sus esfuerzos por resolver los anagramas. No obstante, si los observadores creían que el modelo tenía una habilidad igual a la de ellos, su autoeficacia y persistencia menguaba (Zimmerman, 1995). Debemos señalar que la influencia de la experiencia vicaria en la Autoeficacia para la Realización de Tesis podría verse ejemplifi- 
cada en el caso de la universidad $\mathrm{N}^{\mathrm{o}} 3$. Aquí apreciamos que, desde el periodo 1998-2003 promociones seguidas de estudiantes de psicología no realizan tesis.

Cabe agregar que, dado que la autoeficacia es una buena predictora del comportamiento académico, se puede inferir que un elevado porcentaje de estudiantes de estas tres universidades no elegirá realizar una tesis de investigación, si es que desean optar por la licenciatura universitaria. La afirmación de la autoeficacia como gravitante para el comportamiento académico es sustentada por estudios de Schunk (2001), en los cuales incrementó las creencias de eficacia de estudiantes mediante tácticas como el modelaje y el entrenamiento para plantear estrategias; además de proporcionar recompensas y retroalimentación. Schunk pudo apreciar, después de la capacitación, un aumento en el desempeño y el éxito académico de estos estudiantes (Pajares \& Schunk, 2001).

También se puede explicar que al menos un buen porcentaje de estudiantes no optará por la realización de una tesis, si consideramos la tesis como una actividad compleja y en la que son necesarios altos grados de persistencia. Siguiendo a Bandura (1995) podemos afirmar que los estudiantes con una elevada creencia en sus capacidades personales elegirán tareas con altos niveles de desafío.

Otra posible explicación del bajo nivel de Autoeficacia para la Realiza- ción de una Tesis sería la baja autoeficacia para la enseñanza de los profesores encargados de los cursos relacionados con la investigación, claves para la estructuración de una tesis, debido a que los profesores seguros de su capacidad para enseñar elevan la creencia de control de sus alumnos, su capacidad para autodirigirse e intereses intrínsecos; mientras que los profesores con baja creencia en su capacidad para enseñar disminuyen la autoeficacia de sus alumnos para lograr un rendimiento exitoso. Da soporte a este planteamiento una investigación llevada a cabo por Ashton y Webb (1986), en la que las creencias de los profesores en su eficacia para enseñar predijeron el éxito académico de sus estudiantes en el transcurso del año, sin importar la habilidad académica de los estudiantes al inicio del año (Bandura, 1995).

En lo concerniente al nivel de Disposición para la Realización de una Tesis, los resultados nos hacen ver que, efectivamente, existe un nivel superior al moderado de Disposición para la Realización de una Tesis. Esta afirmación se cumple en todos los factores que componen esta variable. Por consiguiente, se podría concluir, en primer lugar, que los estudiantes, en general, consideran que la realización de una tesis es importante para su formación académica.

En segundo lugar, en opinión de los alumnos el medio universitario promueve la realización de tesis, poniendo 
a su disposición los elementos necesarios para concretarla: asesores eficaces, bibliografía e instrumentos adecuados (test, escalas).

$\mathrm{Y}$ en tercer lugar, los estudiantes están convencidos de que una tesis les resulta útil para conseguir futuros logros profesionales, tanto en el campo académico, posibilitando el acceso a estudios de grados superiores (maestrías, doctorados) dentro o fuera del país, como en el campo laboral, al facilitar la obtención de un trabajo.

Se puede afirmar finalmente, los estudiantes piensan que tienen una formación teórico-metodológica lo suficientemente adecuada para desarrollar una tesis.

\section{REFERENCIAS}

Alarcón, R. (1991). Métodos y diseños de investigación del comportamiento. Lima: Universidad Cayetano Heredia.

Anastasi, A. \& Urbina S. (1998). Tests psicológicos (7a. ed.). México D.F.: Prentice Hall.

Aron, A. \& Aron, E. (2001). Estadística para psicología (2a. ed.). Buenos Aires: Prentice Hall.

Ausubel, D. P. (1995). Psicología educativa. Un punto de vista cognoscitivo. México D.F.: Trillas.

Bandura, A. (1995). Exercise of personal and collective efficacy. En: Bandura, A. (Ed.), Self-efficacy in changing societies (pp. 1-45). Cambrigde, Massachussets: Cambridge University Press.

Bandura, A. (2001). Guía para la construc- ción de Escalas de autoeficacia. Córdoba (Argentina): Universidad Nacional de Córdoba. [en línea]. $<$ http://www.google.com.pe/search?q= cache:LwoZEUC_ORYJ:www.revistaevaluar.com.ar/effguideSpanish.htm $+\mathrm{g}$ $\mathrm{u}$ por cientoC3 por cientoADa+ para + la + construcci por cientoC 3 por cientoB3 $n+$ de + escalas + de + autoeficacia + universidad $+d e+c$ por cientoC3 por cientoB3rdoba\&hl=es $>$

Bandura, A. (1997). Self-efficacy. The exercise of control. Nueva York: W. H. Freeman and Company.

Barberá, E. \& Cantero, Ma. J. (1996). Motivación de logro y categorización de género. En: Garrido, I. (Ed.). Psicología de la motivación (pp. 287-307). Madrid: Síntesis.

Barberá, E. \& Molero, C. (1996). Motivación social. En: Garrido, I. (Ed.). Psicología de la motivación (pp. 163-194). Madrid: Síntesis.

Campos, A. (1993). Manual para la estructuración de la tesis universitaria. Lima: Unifé.

Cattell, R. \& Kline, P. (1982). Análisis científico de la personalidad y la motivación. Madrid: Pirámide.

Chóliz, M. (1995). Prácticas de psicología de la motivación y de la emoción. Valencia: PromoLibro.

De Santamaría, Ma. C. (1991). Motivación de logro. En: Mankeliunas, M. (Ed.). Psicología de la motivación (pp. 177200). México: Trillas.

Hackett, G. (1995). Self-efficacy in career choice and educational development. En: Bandura, A. (Ed.). Self-efficacy in Changing Societies (pp. 232-258). Cambridge, Massachussetts: University 
of Cambridge Press.

Hellriegel, D. (1998). Comportamiento organizacional. (8a. ed.). México: Thomson.

Hernández, S. \& Fernández, C. (1995). Metodología de la investigación. México: McGraw-Hill.

McClelland, D. (1967). The achieving society. Nueva York: The Free Press.

Merani, A. Diccionario de psicología. México: Grijalbo.

Myers, D. (2000). Psicología social. (6a. ed.). Bogotá: McGraw-Hill.

Nunnally, J. \& Bernstein, I. (1995). Teoría psicométrica. (2a. ed.). México: McGraw-Hill.

Pajares, F. (1996). Self-efficacy Beliefs in Academic Settings. En: Riding, R. \& Rayner, S. (Eds). Perception (pp. 239266). Londres: Ablex Publishing. [en línea].<http://www.emory.edu/ EDUCATION/mfp/efftalk.html>

Pajares, F. (2002). Overview of social cognitive theory and self-efficacy. Atlanta: Emory University. [en línea]. $<$ http://www.emory.edu/EDUCATION/mpf/eff.html>

Pajares, F. (1996). Assesing self-efficacy beliefs and academic outcomes: The case for specificity and correspondence. Annual Meeting of the American Educational Research Association. Nueva York. [en línea]. <http://www.emory. edu/EDUCATION/mfp/aera2.html>

Pajares, F. \& Schunk, D. (2001). Self belief and school success: Self-efficacy, self concept, and school achievement. [en línea]. <http://www.emory.edu/EDUCATION/mfp/PajaresSchunk2001.htm>

Papalia, D. \& Wendkos S. (1987). Psicolo- gía. México: McGraw-Hill.

Pontificia Universidad Católica del Perú. Estudios de la especialidad de psicología. [en línea]. <http://www.pucp. edu.pe/fac/llcchh/psicologia.htm>

Pozo, A. (2003). Personalidad, autocontrol, autorregulación y autoeficacia en el ámbito familiar. En: Polaino, A. (Ed). Fundamentos de desarrollo de la personalidad (pp. 408-424). Madrid: Rialp.

Santrock, J. W. (2002). Psicología de la educación. México: McGraw-Hill.

Thornberry, G. (2003). Relación entre Motivación de Logro Académico y Rendimiento Académico en alumnos de colegios limeños de diferente gestión. Tesis para obtener el título de licenciado en psicología. Lima: Universidad de Lima.

Universidad de Lima (2003). Presentación de la carrera de psicología. [en línea]. $<$ http://fresno.ulima.edu.pe/sf/sf_bd50 01.nsf/default/car?OpenDocument\&dn $=1.1 \mathrm{P}$

Universidad Femenina (2003). Presentación de la carrera de psicología. [en línea]. <http://www.unife. edu.pe/psi$\mathrm{col} / \mathrm{psicol} . \mathrm{html}>$

Universidad Ricardo Palma (2003). Presentación de la carrera de psicología. [en línea]. <http://www.urp. edu.pe/>

Woolfolk, A. E. (1996). Psicología educativa. (6a. ed.). México: Prentice Hall.

Zimmerman, B. J. (1995). Self-efficacy and educational development. En: Bandura, A. (Ed.). Self-efficacy in changing societies (pp. 202-231). Cambridge, Massachusetts: University of Cambridge Press. 


\section{APÉNDICE 1}

\section{Cuestionario 1 \\ Sobre la realización de una tesis}

\section{Instrucciones:}

Este cuestionario contiene algunos enunciados sobre ciertas situaciones con respecto a una tesis. Léelos cuidadosamente e indica en qué grado estás seguro de poder realizar una tesis en cada situación.

Marca en la hoja de respuestas con un aspa la letra que mejor te describa:

$\begin{array}{ll}\text { S: } & \text { Siempre } \\ \text { F: } & \text { Frecuentemente } \\ \text { AV: } & \text { A veces } \\ \text { RV } & \text { Rara vez } \\ \text { N: } & \text { Nunca }\end{array}$

Recuerda que no hay respuestas correctas o incorrectas. Trabaja rápidamente y no emplees mucho tiempo en cada pregunta. Si deseas hacer una corrección, borra completamente el aspa y vuelve a escribir otra en el lugar de la nueva respuesta. Trata de responder de acuerdo a lo que haces en estas situaciones y asegúrate de responder todas las oraciones. Si has terminado, revisa que hayas marcado todos los enunciados en la hoja de respuestas y pasa al siguiente cuestionario.

Yo puedo realizar una tesis de investigación:

1. Cuando me siento cansado de estudiar

2. Cuando estoy presionado por el estudio o el trabajo

3. Durante o después de haber experimentado problemas personales

4. Cuando tengo compromisos sociales

5. Cuando tengo mucho trabajo que hacer en casa

6. Cuando tengo que buscar mucha bibliografía

7. Cuando tengo que buscar muchos materiales. Como, por ejemplo, test, escalas, entre otros

8. Cuando mi familia y mis amigos no me apoyan

9. Luego de experimentar problemas familiares

10. Cuando tengo problemas económicos

11. Cuando estoy deprimido

12. Cuando me siento ansioso

13. Cuando hay otras actividades interesantes para hacer

14. Cuando no recibo el apoyo de mi asesor de tesis

\begin{tabular}{ll}
\hline Factor & ítems \\
\hline Autoeficacia Personal-Social & $3,4,5,8,9,10,11,12,13,14$ \\
Autoeficacia Académica & $1,2,6,7$ \\
\hline
\end{tabular}




\section{APÉNDICE 2}

\section{Cuestionario 2 \\ De hábitos de estudio}

\section{Instrucciones}

Este cuestionario contiene algunas frases respecto a tus hábitos de estudio. Lee cada una de ellas y marca con aspa, en la hoja de respuestas, la letra que mejor te describa.

S: Siempre

F: $\quad$ Frecuentemente

AV: A veces

RV Rara vez

$\mathrm{N}$ : Nunca

Recuerda que no hay respuestas correctas o incorrectas. Trabaja rápidamente y no emplees mucho tiempo en cada ítem. Si deseas hacer una corrección, borra completamente el aspa y vuelve a escribir otra en el lugar de la nueva respuesta. Trata de responder de acuerdo a cómo realmente actúas o actuarías. Asegúrate de responder todas las oraciones. Si has terminado, revisa que hayas marcado todos los enunciados en la hoja de respuestas y pasa al siguiente cuestionario.

1. Preparo mis exámenes con anticipación

2. Reviso los errores de mis exámenes

3. Busco temas nuevos para mis trabajos

4. El grado de dificultad de un examen determina la nota que yo obtenga

5. Analizo bien un área, antes de escogerla como tema de trabajo

6. Cuando tengo que hacer un trabajo exigente, calculo el tiempo disponible

7. Contesto a las preguntas del profesor con respuestas seguras, antes de arriesgarme con algo diferente

8. Estudio un día antes de los exámenes

9. Hago ensayos de mis exposiciones, pidiendo la opinión de mis compañeros

10. Elijo un tema conocido, con gran cantidad de información, en vez de uno nuevo poco investigado

11. Cuando desapruebo un examen soy el único responsable

12. El trabajo que me propongo hacer está dentro de mi capacidad

13. Cuando tengo que hacer un trabajo exigente, me organizo

14. Me pongo nervioso en un examen

15. Me molesto cuando el profesor realiza preguntas obvias

16. Estudio solo para aprobar los cursos

17. Busco las respuestas correctas a mis errores en los exámenes

18. Escojo un tema entre aquellos que se han investigado más

19. Cuando obtengo una buena calificación en un examen es porque el examen es fácil

20. Analizo la disponibilidad de información antes de realizar un trabajo

21. Me gustan los trabajos que representen un reto

22. Lo enseñado en clases me llama poco la atención

23. Me inhibo de expresar conclusiones fuera de lo común, acerca de lo enseñado en clase 
24. Preparo mis trabajos con anticipación

25. Me impaciento si el profesor demora en entregar las notas de un examen

26. Busco aspectos novedosos a los temas ya conocidos

27. En una situación de examen complicado suelo controlar mi ansiedad

28. Si un trabajo es posible de realizar, pero presenta alta dificultad, lo dejo de lado

29. Prefiero los trabajos que exigen un esfuerzo mayor

30. Me es indiferente desaprobar algunos cursos

31. Me molestan los profesores que, en clase, demandan la aplicación de lo que han enseñado a contextos diferentes

32. Entrego mis trabajos individuales a tiempo

33. Si una calificación me parece inadecuada, pregunto al profesor por la respuesta correcta

34. Si me dan un tema ampliamente conocido, intento presentarlo de manera que sea interesante

35. La nota de un examen es coherente con el esfuerzo que realizo

36. Cuando preparo una exposición, calculo el tiempo en relación a la información que voy a exponer

37. Realizo con éxito un trabajo a pesar de que presente alta dificultad

38. Los temas que he desarrollado en mis trabajos me han motivado

39. Me molestan los profesores que, en clase, demandan pensar en respuestas no convencionales a las preguntas que hacen

40. Consulto el mínimo número de fuentes bibliográficas para hacer mis trabajos

41. Reviso las correcciones hechas a mis trabajos

42. Presento mis exposiciones de una manera interesante

43. Reviso mis trabajos, antes de entregarlos, para corregir errores

44. Pongo mucha atención a lo que el profesor desea de una exposición

45. Al realizar una exposición difícil calculo el tiempo suficiente para investigar

46. Aplico las sugerencias de mis profesores a mis trabajos

47. Si la nota de un trabajo es ligeramente menor a la esperada, pido explicación

48. En mis exposiciones utilizo técnicas que salen fuera de lo común

49. La calificación alta de un trabajo depende, en su mayor parte, de mi esfuerzo

50. Cuando preparo una exposición busco la información necesaria para que sea calificada con nota aprobatoria

51. Cuando hago una exposición difícil tomo el tiempo necesario para prepararla adecuadamente

52. Coincido con las ideas de mis profesores

53. Pido ampliación de tiempo para entregar mis trabajos

54. Pongo atención a los comentarios hechos por mis profesores sobre los trabajos que presento

55. Mis exposiciones despiertan curiosidad en mis compañeros

56. La nota baja, en un trabajo, es producto de la alta exigencia de un profesor

57. Me desagradan los grupos que sólo eligen trabajos fáciles

58. Hago exposiciones en las que, debido al tema, tengo que prepararme bastante

59. Asisto puntualmente a las clases

60. Preparo mis exposiciones un día antes de la presentación

61. Pido asesoría para asegurarme de que estoy realizando bien un trabajo

62. En mis exposiciones empleo materiales que llamen la atención

63. En una exposición, la calificación solo depende de la opinión del profesor

64. Al hacer un trabajo grupal prefiero juntarme con cualquier persona

65. Para las exposiciones en temas difíciles me documento bastante 
66. Consulto la bibliografía antes de ir a clase

67. Pido que posterguen la fecha de presentación de mis exposiciones

68. Me interesa la opinión de mis compañeros de grupo sobre mi desempeño

69. En mis exposiciones me centro más en el contenido del tema que en los detalles de presentación

70. Soy el responsable de la nota que obtengo en una exposición

71. Cuando trabajo en grupo, el tema lo hago de forma aceptable

72. Me interesa que el tema grupal tenga consenso, para hacer un buen trabajo

73. Devuelvo rápidamente los apuntes prestados a mis compañeros

74. Me documento conscientemente para realizar mis exposiciones

75. Acepto de mala gana las críticas grupales, incluso si estoy trabajando mal

76. En mi grupo hago tareas novedosas

77. Cuando expongo un trabajo, la ansiedad disminuye mi desempeño

78. Mantengo un clima grupal agradable para desarrollar un mejor trabajo

79. Trato de atender lo que dicen mis profesores en clase

80. Hago ensayos previos a mis exposiciones

81. Cuando estoy trabajando mal, desearía que mi grupo me lo dijera

82. Prefiero los grupos que hacen trabajos fuera de lo común

83 El grado de mi esfuerzo determina la nota en una exposición

84. Para hacer un trabajo grupal me reúno con personas que son exigentes

85. Me distraigo en clases

86. Analizo y comento los trabajos con mi grupo antes de entregarlos

87 Preparo mis exposiciones con tiempo.

88. En los trabajos grupales me da igual el tema que se elija

89. El éxito de un trabajo grupal depende de las otras personas del grupo

90. Hago que las otras personas del grupo asuman mayores responsabilidades

91. Reviso mis apuntes antes de ir a clases

92. Hago los trabajos grupales con mis amigos, aunque ellos tengan un bajo rendimiento

93. En mi grupo, propongo buscar aspectos más interesantes que los temas de siempre

94. Cuando un trabajo es bien evaluado, gran parte del mérito es mío

95. Voy a grupos donde sé que los otros asumirán más responsabilidades

96. Si tengo una duda, intervengo en clase

97. Hago los trabajos grupales con personas de alto rendimiento académico

98. Me interesan los comentarios que generan mis exposiciones entre los compañeros

99. En mis trabajos grupales, utilizo bibliografía actualizada y novedosa

100. Si un trabajo grupal es mal evaluado, los demás son los responsables del fracaso

101. Pido prestados los apuntes de otros para estudiar

102. Cuando escojo un grupo, me aseguro de que todos los integrantes sean responsables

103. Presto poca atención a los comentarios del profesor sobre mis exposiciones

104. Mis intervenciones en clases son originales

105. Al momento de trabajar en grupo, controlo mi impaciencia

106. Llego tarde a mis clases

107. Si considero que la nota de una exposición es injusta, consulto al profesor por la razón de esa evaluación 


\begin{tabular}{ll}
\hline Factor & \multicolumn{1}{c}{ Ítem } \\
\hline Búsqueda activa de éxito académico & $\begin{array}{l}8,16,24,32,40,53,60,67,74,79,85,87,91,92,96,97, \\
101,106\end{array}$ \\
Retroalimentación & $2,9,17,33,41,47,54,61,68,75,81,86,98,103$ y 107 \\
Originalidad & $3,7,10,18,26,34,39,42,48,55,62,76,82,93,99$ y 104 \\
Atribución personal del éxito y & $11,19,35,43,49,56,63,70,83,89,100$ y 105 \\
$\quad$ regulación de tareas & \\
Metas altas realistas & $6,13,29,21,37,45,51,58,65,72,78,84,90$ y 95 \\
Deseabilidad social-mentiras & $14,22,30,38,46,52,59,66,73$ \\
\hline
\end{tabular}

\section{APÉNDICE 3}

\section{Cuestionario 3 \\ Sobre el interés para realizar una tesis de investigación}

\section{Instrucciones}

Este cuestionario contiene algunas frases respecto de la realización de una tesis de investigación. Lee cada una de ellas y, en la hoja de respuestas, marca con un aspa la opción que crees expresa mejor lo que piensas:

DA: De acuerdo

I: Indeciso

ED: En desacuerdo

TD: Totalmente en desacuerdo

Recuerda que no hay respuestas correctas o incorrectas. Trabaja rápidamente y no emplees mucho tiempo en cada ítem. Si deseas hacer una corrección, borra completamente el aspa y vuelve a escribir otra en el lugar de la nueva respuesta. Trata de responder de acuerdo con lo que realmente piensas o sientes. Asegúrate de responder todos los enunciados

1. Realizar la tesis tiene una importancia personal

2. Poseo una motivación personal para realizar una tesis

3. Preparar una tesis es útil para conseguir trabajo

4. Tengo posibilidades personales para realizar una tesis

5. Tengo conocimientos teóricos adecuados para realizar una tesis

6. Tengo acceso a los asesores de mi facultad para realizar una tesis

7. Tengo acceso a la lectura de tesis de otras universidades para realizar una investigación

8. Es importante para la universidad que yo realice la tesis

9. Necesito hacer la tesis para obtener el título universitario o la licenciatura

10. Mis profesores me motivan a realizar una tesis

11. Realizar una tesis es útil para conseguir una beca de estudios 
12. Mi facultad brinda facilidades para la realización de una tesis

13. En los cursos de metodología he recibido una formación que me permite realizar una tesis

14. En mi facultad, los asesores de tesis son eficaces

15. Tengo acceso a la lectura de tesis en mi facultad para realizar una investigación

16. Hacer una tesis es importante para mi formación en investigación

17. Necesito realizar una tesis para ejercer mi carrera

18. Mi facultad promueve la realización de tesis

19. Hacer una tesis es útil para postular a estudios en el exterior

20. En mi facultad es complicado realizar una tesis

21. Tengo una necesidad personal de realizar la tesis

22. He recibido una formación académica que me permite realizar una tesis

23. En mi facultad existe facilidad para designar asesores de tesis

24. En este momento puedo proponer algunos temas de interés para una tesis

25. Realizar una tesis es importante para mi futuro trabajo

26. Necesito realizar una tesis para familiarizarme con los procesos de investigación

27. Deseo realizar una tesis para profundizar un tema de mi interés

28. Realizar una tesis es útil para postular a una maestría o a un doctorado

29. Mis conocimientos metodológicos me permiten realizar una tesis

30. Tengo acceso a bibliografía especializada para realizar una tesis

31. Dispongo de los medios económicos para realizar una tesis

32. Tengo habilidad para proponer temas de interés para una tesis

33. Realizar una tesis es importante para definir una fuente de interés profesional

34. Necesito realizar una tesis para afianzar mi formación académica

35. Deseo hacer una tesis para proporcionar aportes en mi área del conocimiento

36. La realización de una tesis es útil para la obtención de prestigio, en un área del conocimiento

37. Actualmente dispongo de tiempo para la realización de la tesis

38. Tengo acceso a la bibliografía necesaria para realizar una tesis

39. La inversión económica en una tesis está justificada

40. Es importante realizar una tesis en mi área de especialización (clínica, organizacional, social, educativa)

41. Es útil realizar una tesis en mi área de especialidad (clínica, organizacional, educativa, social)

42. Al finalizar mis estudios de pregrado dispondré de tiempo suficiente para realizar una tesis

43. Es importante hacer una tesis para seguir estudios de postgrado

44. Tengo acceso al uso de instrumentos para realizar una tesis. Como, por ejemplo, test y escalas

\begin{tabular}{ll}
\hline Factor & \multicolumn{1}{c}{ Ítem } \\
\hline Formación académica & $1,16,17,21,24,25,26,27,31,33,34,35,39,40,41$, \\
& 42,43 \\
Facilidades académicas & $6,7,8,10,12,14,15,18,20,22,23,30,38,44$ \\
Utilidad futura & $3,9,11,19,28,36$ \\
Confianza académica para la tesis & $2,4,5,13,29,32$ \\
\hline
\end{tabular}

